\title{
Cavity voltage phase modulation to reduce the high-luminosity Large Hadron Collider rf power requirements
}

\author{
T. Mastoridis \\ California Polytechnic State University, San Luis Obispo, California 93407, USA \\ P. Baudrenghien and J. Molendijk \\ CERN, Geneva 1211, Switzerland
}

(Received 21 March 2017; published 10 October 2017)

\begin{abstract}
The Large Hadron Collider (LHC) radio frequency (rf) and low-level rf (LLRF) systems are currently configured for constant rf voltage to minimize transient beam loading effects. The present scheme cannot be extended beyond nominal LHC beam current $(0.55 \mathrm{~A} \mathrm{dc})$ and cannot be sustained for the highluminosity (HL-LHC) beam current $(1.1 \mathrm{~A} \mathrm{dc})$, since the demanded power would exceed the peak klystron power. A new scheme has therefore been proposed: for beam currents above nominal (and possibly earlier), the voltage reference will reproduce the modulation driven by the beam (transient beam loading), but the strong rf feedback and one-turn delay feedback will still be active for loop and beam stability. To achieve this, the voltage reference will be adapted for each bunch. This paper includes a theoretical derivation of the optimal cavity modulation, introduces the implemented algorithm, summarizes simulation runs that tested the algorithm performance, and presents results from a short LHC physics fill with the proposed implementation.
\end{abstract}

DOI: $10.1103 /$ PhysRevAccelBeams.20.101003

\section{INTRODUCTION}

The high currents employed in modern light sources and circular accelerators lead to strong coupling of the bunch motion with the cavity impedance. The rf system keeps the voltage constant in amplitude and phase to achieve two objectives. First, it compensates the beam loading and thus achieves beam longitudinal stability. Second, it maintains the bucket area unchanged so that there is no effect on bunch emittance and beam loss. This is achieved by a modulation of the generator drive. These modulations scale with the beam current and lead to increased demanded power from the klystrons, and eventually to saturation. As a result, uncompensated beam loading effects in the LHC are really small due to the action of the strong rf feedback and one-turn delay feedback. The resulting cavity voltage has an amplitude modulation less than $1 \%$, whereas the phase modulation is less than $1^{\circ}$, as shown in Fig. 1. This operational scheme maintains a constant cavity voltage in both amplitude and phase by modulating the klystron drive (in amplitude and phase), as shown in Fig. 2.

This scheme though comes at the expense of klystron forward power, especially during the transition between the beam and no-beam segments. At least $200 \mathrm{~kW}$ of klystron

Published by the American Physical Society under the terms of the Creative Commons Attribution 4.0 International license. Further distribution of this work must maintain attribution to the author(s) and the published article's title, journal citation, and DOI. forward power would be necessary at nominal intensity (2808 bunches, $1.1 \times 10^{11}$ protons/bunch). The klystrons saturate at $300 \mathrm{~kW}$, but a margin is required to maintain the rf feedback gain. Figure 2 shows the instantaneous power in the LHC with 2244 bunches and $\approx 1.1 \times 10^{11}$ protons/bunch. The big transients correspond to all the beam pattern gaps used for that fill: $225 \mathrm{~ns}$ due to the proton synchrotron (PS) kicker rise time, $900 \mathrm{~ns}$ due to the super proton synchrotron (SPS) kicker rise time, and finally $6.85 \mu$ s due to the LHC abort gap. The $300 \mathrm{~kW}$ klystron power will not be sufficient for beam intensity much above the nominal LHC parameters and definitely not for the high-luminosity LHC (HL-LHC, $2.2 \times 10^{11}$ protons/bunch) [1].

Boussard proposed a solution to this limitation in 1991 [2]. This method tries to keep the klystron drive constant (in amplitude and phase) by modulating the cavity reference in anticipation of the beam, so that the rf loop-and thus the klystron-does not try to reduce the beam loading effects in the cavity. In other words, the cavity voltage is modulated periodically in both amplitude and phase, so that the klystron drive is constant. This method maintains the strong rf feedback and one-turn delay feedback (OTFB) though, with the corresponding positive effects for longitudinal stability. This algorithm was implemented in simulations $[3,4]$ and during the first LHC run $[5,6]$ with promising results. Figures 3 and 4 show the resulting cavity and klystron signals. It is clear that with this scheme the klystron modulation is significantly reduced, but now the cavity voltage is modulated in both amplitude and 

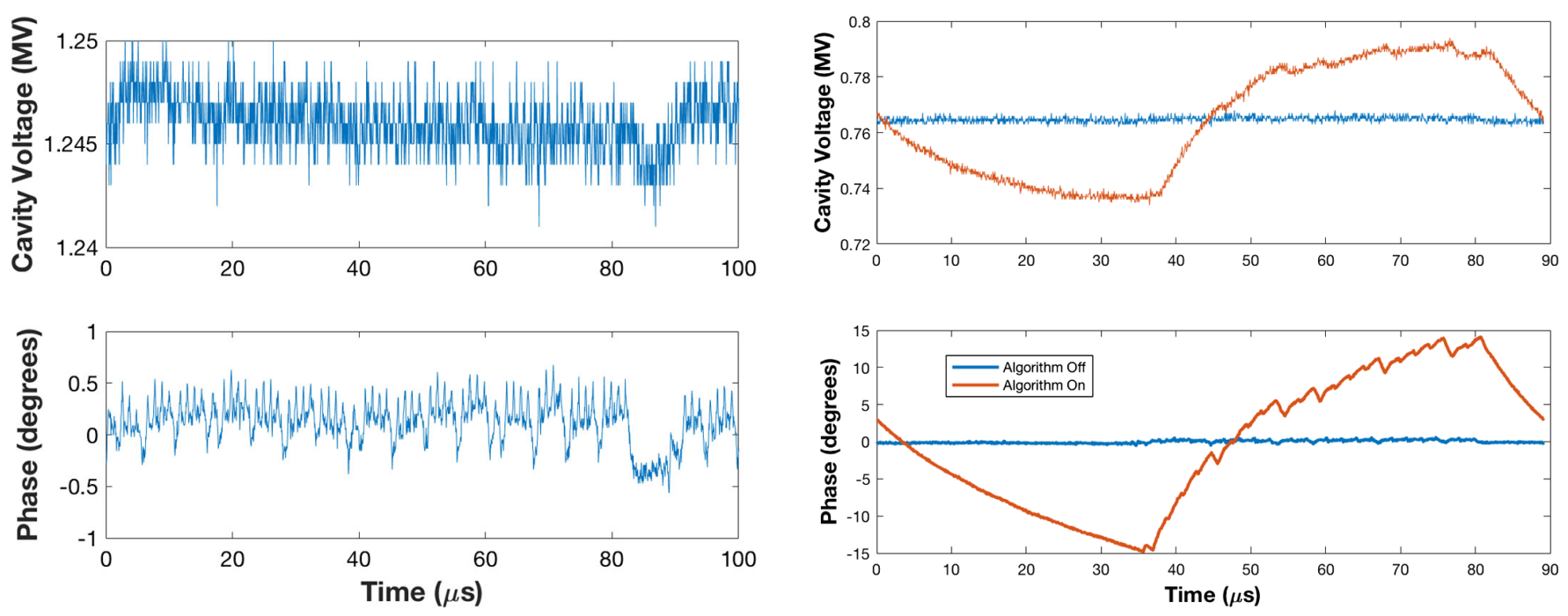

FIG. 1. Cavity voltage with 2244 bunches, $1.2 \times 10^{11}$ protons/ bunch, $25 \mathrm{~ns}$ spacing, $6.5 \mathrm{TeV}$.

phase, with negative consequences for beam stability. Furthermore, the required klystron power was reduced, but did not achieve the expected minimum power levels, in neither the average power over a turn nor on the transients. A similar technique was implemented at PEP-II [7].

It will be shown mathematically in Sec. II that keeping the klystron drive constant is not the optimal strategy. The alternative optimal scheme will be presented in this work, as well as validating results from simulations and LHC tests. This alternative scheme keeps the magnitude of the klystron drive almost constant and maintains very good regulation of the cavity voltage amplitude. To achieve this, it imposes the same phase modulation on the klystron and cavity, as will be shown below.
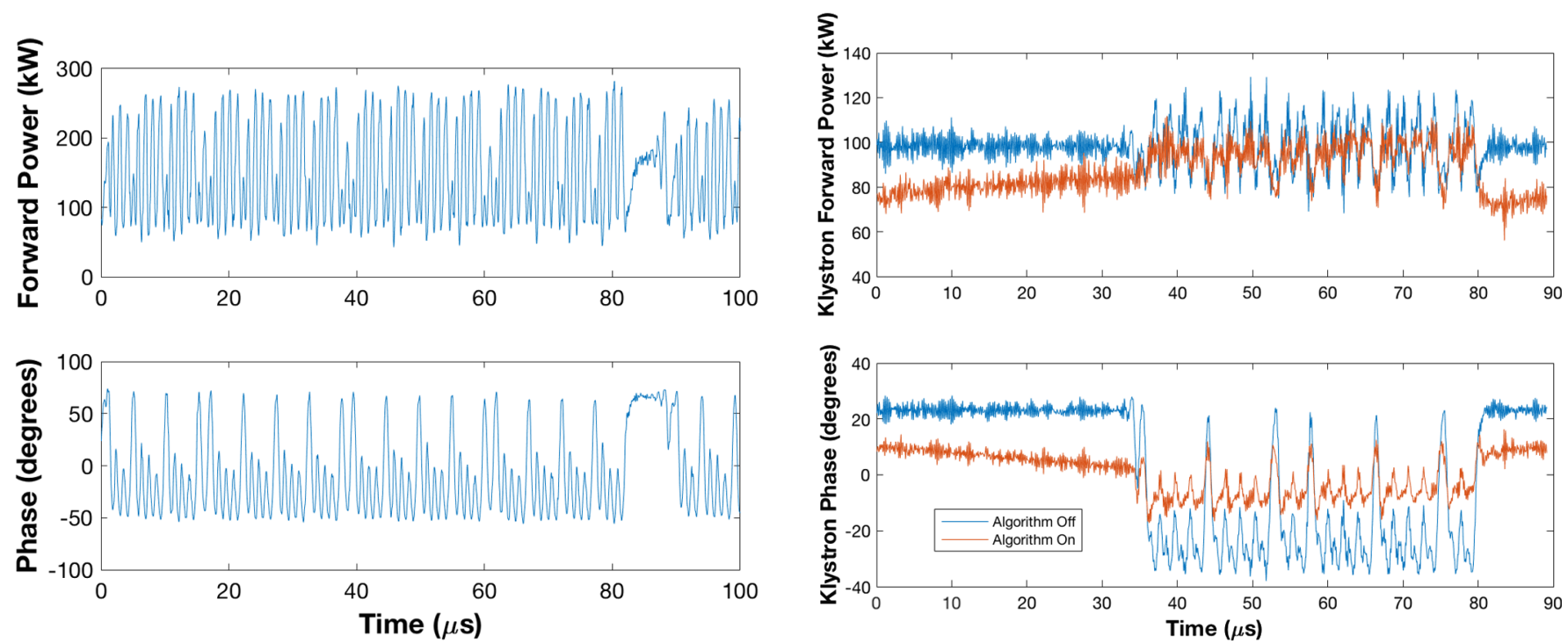

FIG. 2. Klystron power with 2244 bunches, $1.2 \times 10^{11}$ protons/ bunch, $25 \mathrm{~ns}$ spacing, $6.5 \mathrm{TeV}$.

FIG. 3. Cavity voltage with 654 bunches, 50 ns spacing, $450 \mathrm{GeV}$.

Section II introduces a theoretical derivation of the optimal algorithm for klystron power minimization. The actual algorithm implementation is described in Sec. III. The algorithm was tested in simulations (Sec. IV) and in the LHC (Sec. V). After these successful tests, the algorithm was also tested while the LHC detectors were collecting data in $6.5 \mathrm{TeV}$ proton physics to evaluate any possible detrimental effects on luminosity (Sec. VI). Finally, Appendix A presents a technical description of the algorithm implementation in the hardware/firmware and Appendix B includes an LHC (Table I) and HL-LHC (Table II) RF parameter table.

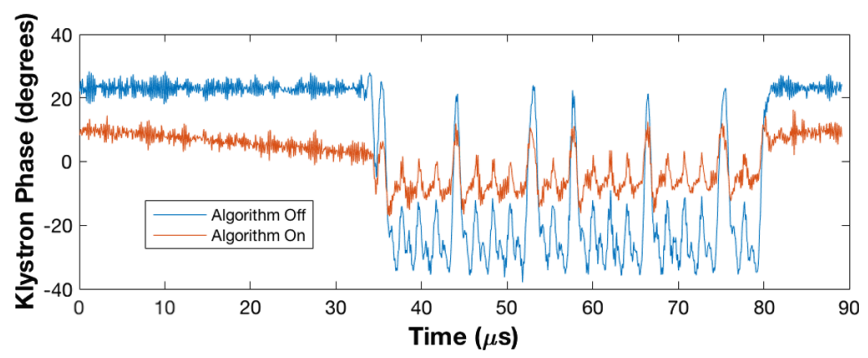

FIG. 4. Klystron power with 654 bunches, 50 ns spacing, $450 \mathrm{GeV}$. 


\section{THEORETICAL MODEL AND OPTIMAL ALGORITHM}

The proposed implementation will keep the voltage amplitude constant and will cancel any noise induced perturbations, as the present scheme, thus keeping the bucket area constant. In addition though, it will accept a phase modulation $\varphi(t)$ of the voltage reference phase which will track the beam induced perturbations, thus maintaining longitudinal stability but also reducing klystron power requirements.

The cavity voltage (after demodulation by the rf frequency) is then

$$
V(t)=V_{o} e^{j \varphi(t)}
$$

where the phase modulation $\varphi(t)$ is periodic at the revolution frequency. The voltage is kept constant to continue providing single and multibunch longitudinal stability. Assuming $180^{\circ}$ stable phase (synchrotron above transition: bunch center on the falling zero crossing of the $\mathrm{rf}$ voltage), the beam current $I_{b}(t)$ [8] is given by

$$
I_{b}(t)=i_{b}(t) e^{j\left[\varphi(t)-\frac{\pi}{2}\right]}=-j i_{b}(t) e^{j \varphi(t)},
$$

where $i_{b}(t)$ is a scalar representing the beam current modulation. This expression holds for a single cavity. For several cavities, the beam current is in quadrature with the vectorial voltage sum. In this work, it is assumed that all the cavities are aligned, as is done in the LHC, and thus the expression holds for all cavities. $V_{o}$ is the voltage per cavity.

Following [9], the generator current transformed at the cavity gap $I_{g}(t)$ is then given by

$$
I_{g}(t)=\frac{V(t)}{2 R / Q}\left[\frac{1}{Q_{L}}-2 j \frac{\Delta \omega}{\omega}\right]+\frac{d V(t)}{d t} \frac{1}{\omega R / Q}+\frac{I_{b}(t)}{2},
$$

where $\omega$ is the $\mathrm{rf}$ frequency in $\mathrm{rad} / \mathrm{s}, \Delta \omega$ the cavity detuning, $Q_{L}$ the loaded cavity $Q$ factor (varied from 20000 at injection to 60000 in physics), and $R / Q$ is the cavity-shape constant equal to $45 \Omega$ for the LHC cavities [10]. Thus,

$I_{g}(t) e^{-j \varphi(t)}=\frac{V_{o}}{2 R / Q Q_{L}}+j\left[-\frac{V_{o}}{R / Q} \frac{\Delta \omega}{\omega}+\frac{V_{o} \frac{d \varphi}{d t}}{\omega R / Q}-\frac{1}{2} i_{b}(t)\right]$.

As a result, the instantaneous required power $P(t)$ as derived in [9] is

$$
\begin{aligned}
P(t)= & \frac{1}{2} R / Q Q_{\mathrm{ext}}\left|I_{g}(t)\right|^{2} \\
= & \frac{V_{o}^{2}}{8 R / Q Q_{L}}+\frac{1}{2} R / Q Q_{L}\left[-\frac{V_{o}}{R / Q} \frac{\Delta \omega}{\omega}\right. \\
& \left.+\frac{V_{o} \frac{d \varphi}{d t}}{\omega R / Q}-\frac{1}{2} i_{b}(t)\right]^{2},
\end{aligned}
$$

where $Q_{\text {ext }} \approx Q_{L}$ since the LHC cavities are superconducting. The instantaneous required power is minimized when the term in brackets is equal to zero. In that case, the required power is independent of beam current and equal to the power without beam. The required power can then be further reduced by increasing $Q_{L}$ (the LHC cavities have movable couplers).

The term in brackets can be expressed in terms of the generator current and rf component of the cavity voltage $V(t)$ :

$\left[-\frac{V_{o}}{R / Q} \frac{\Delta \omega}{\omega}+\frac{V_{o} \frac{d \varphi}{d t}}{\omega R / Q}-\frac{1}{2} i_{b}(t)\right]=\frac{\operatorname{Im}\left[I_{g}(t) \bar{V}(t)\right]}{V_{o}}=0$,

where $\bar{V}(t)$ is the complex conjugate of $V(t)$. Therefore, the required power is minimum when the generator current and the cavity voltage have identical phase modulation. This result deviates from previous works that aimed at keeping the generator current fixed in both amplitude and phase [1-6].

\section{A. Optimal detuning}

It is possible to rearrange Eq. (2) to calculate the detuning $\Delta \omega$ when the klystron power has been minimized:

$$
\begin{aligned}
& -\frac{V_{o}}{R / Q} \frac{\Delta \omega_{\mathrm{opt}}}{\omega}+\frac{V_{o} \frac{d \varphi}{d t}}{\omega R / Q}-\frac{1}{2} i_{b}(t)=0 \\
& \int_{t}^{t+T_{\mathrm{rev}}}\left[-\frac{V_{o}}{R / Q} \frac{\Delta \omega_{\mathrm{opt}}}{\omega}+\frac{V_{o} \frac{d \varphi}{d u}}{\omega R / Q}-\frac{1}{2} i_{b}(u)\right] d u=0 \\
& -\frac{V_{o}}{R / Q} \frac{\Delta \omega_{\mathrm{opt}}}{\omega} T_{\mathrm{rev}}+\frac{V_{o}\left[\varphi\left(t+T_{\mathrm{rev}}\right)-\varphi(t)\right]}{\omega R / Q} \\
& -\int_{t}^{t+T_{\mathrm{rev}}} \frac{1}{2} i_{b}(u) d u=0,
\end{aligned}
$$

where $T_{\text {rev }}$ is the revolution period. After convergence, the cavity phase is periodic and thus $\varphi\left(t+T_{\text {rev }}\right)=\varphi(t)$. As a result, the optimal detuning is equal to

$\Delta \omega_{\mathrm{opt}}=-\frac{1}{2} \frac{\omega R / Q}{V_{o}} \frac{\int_{t}^{t+T_{\mathrm{rev}}} i_{b}(u) d u}{T_{\mathrm{rev}}}=-\frac{1}{2} \frac{\omega R / Q}{V_{o}} \overline{I_{b}}$,

where $\overline{I_{b}}$ is the average rf component of the beam current. This optimal detuning is defined as "full detuning" and it is the optimal detuning if there are no gaps in the beam pattern and the current is spread evenly along the ring [9]. 


\section{B. Optimal cavity phase modulation}

Inserting the optimal detuning in Eq. (2), it is also possible to calculate the optimal cavity phase modulation:

$$
\begin{aligned}
\frac{d \varphi}{d t} & =\Delta \omega_{\mathrm{opt}}+\frac{\omega R / Q}{2 V_{o}} i_{b}(t) \\
\frac{d \varphi}{d t} & =-\Delta \omega_{\mathrm{opt}} \frac{i_{b}(t)-\overline{I_{b}}}{\overline{I_{b}}} \\
\varphi(t) & =-\Delta \omega_{\mathrm{opt}} \int_{t_{0}}^{t} \frac{i_{b}(u)-\overline{I_{b}}}{\overline{I_{b}}} d u+\varphi\left(t_{0}\right) .
\end{aligned}
$$

The constant $\varphi\left(t_{0}\right)$ is a "free" parameter as power does not depend on a constant phase shift of the cavity phase. But this must be constrained to keep all cavities in phase (for a given ring), and retain the collision point at the detector center. The algorithm imposes a zero phase modulation average over a turn.

In the presence of beam segments and gaps, Eq. (4) leads to a piecewise linear phase modulation. A very important consequence is the linear dependence of the peak-to-peak phase modulation to the abort gap length for a constant total beam current. Small changes in the LHC beam pattern could lead to significant changes in the phase modulation. It should also be noted that the earlier work [2] focused on a fixed klystron drive and resulted in an exponential voltage phase modulation [1], as seen in Fig. 3.

Figure 5 shows the estimated cavity phase modulation for the LHC design report filling pattern and the HL-LHC beam parameters $\left(2.2 \times 10^{11}\right.$ protons per bunch at $\left.7 \mathrm{TeV}\right)$. The peak-to-peak variation is only $111 \mathrm{ps}$ for an $\approx 1 \mathrm{~ns}$ long bunch. Furthermore, since this modulation would be almost

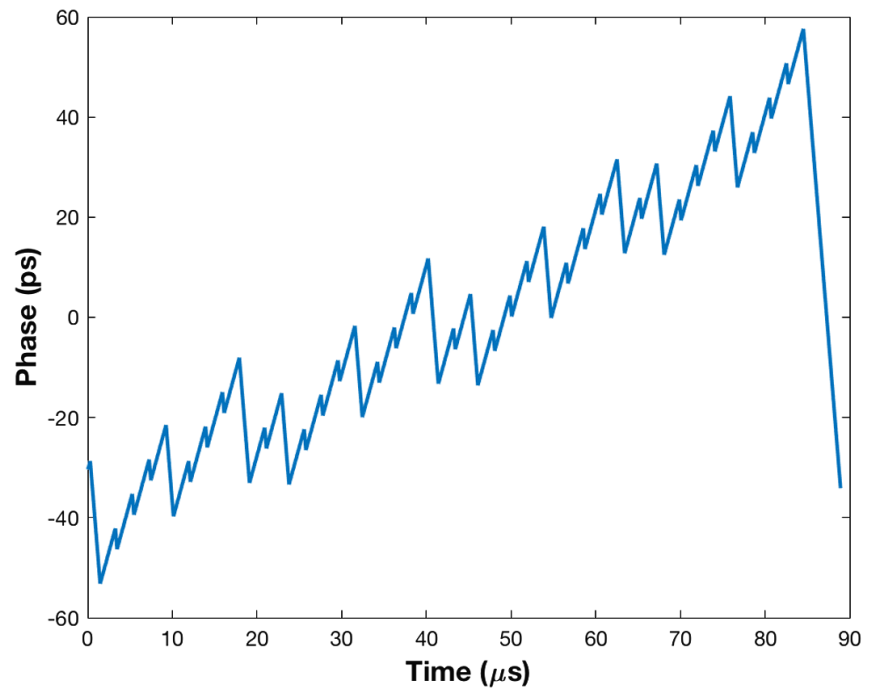

FIG. 5. Calculated cavity phase modulation for HL-LHC filling pattern and beam parameters. 2748 bunches, $2.2 \times 10^{11}$ protons per bunch, $7 \mathrm{TeV}, 16 \mathrm{MV}$ rf voltage, $4.4 \mu$ s long abort gap. The phase is reported in ps with respect to the $400 \mathrm{MHz}$ rf clock. symmetric for the two rings, the collision point would barely shift in the LHC interaction points 1 and 5 (ATLAS and CMS experiments [11]).

\section{ALGORITHM IMPLEMENTATION}

The generator current, cavity reference, and cavity voltage are sampled at $40 \mathrm{MHz}$ in the LHC. The discrete version for Eq. (1) is

$$
\begin{aligned}
P_{n}^{(k)}= & \frac{1}{2} R / Q Q_{L}\left|I_{g_{n}}^{(k)}\right|^{2} \\
= & \frac{V_{o}^{2}}{8 R / Q Q_{L}}+\frac{1}{2} R / Q Q_{L}\left[-\frac{V_{o}}{R / Q} \frac{\Delta \omega}{\omega}\right. \\
& \left.+\frac{V_{o} \dot{\varphi}_{n}^{(k)}}{\omega R / Q}-\frac{1}{2} i_{b_{n}}^{(k)}\right]^{2},
\end{aligned}
$$

where $n$ is the time index and $k$ is the algorithm iteration index.

Using a steepest-descent algorithm [12], the update of the phase modulation becomes

$$
\begin{aligned}
\dot{\varphi}_{n}^{(k+1)} & =\dot{\varphi}_{n}^{(k)}+\alpha \frac{\partial P_{n}^{(k)}}{\partial \dot{\varphi}_{n}^{(k)}} \\
& =\dot{\varphi}_{n}^{(k)}+\alpha \frac{V_{o} Q_{L}}{\omega}\left[-\frac{V_{o}}{R / Q} \frac{\Delta \omega}{\omega}+\frac{V_{o} \dot{\varphi}_{n}^{(k)}}{\omega R / Q}-\frac{1}{2} i_{b_{n}}^{(k)}\right] \\
& =\dot{\varphi}_{n}^{(k)}+\alpha \frac{Q_{L}}{\omega} \operatorname{Im}\left[I_{g_{n}}^{(k)} V_{n}^{(k)}\right]=\dot{\varphi}_{n}^{(k)}+f_{n}^{(k)},
\end{aligned}
$$

where $\alpha$ controls the convergence time leading to a time constant $\tau=-T_{\text {rev }} / \ln (1+\alpha)$ with $T_{\text {rev }}$ the revolution period.

In the actual implementation, $I_{g}$ and $V$ are sampled every $25 \mathrm{~ns}$ for each of the 3564 possible bunch locations at every turn $\left(T_{\text {rev }} \approx 88.9 \mu \mathrm{s}\right)$. Then the error function $f^{(k)}$ and the new $\dot{\varphi}$ values are computed. The parameter $\alpha$ corresponds to the system gain. The gain is set so that the algorithm time constant is about 30 seconds, which is much slower than the synchrotron period $(\approx 50 \mathrm{~ms})$ and the cavity frequency tuning loop time constant $(\approx 1$ second). As a result, any changes to the beam phase are adiabatic.

The $\dot{\varphi}$ values are then integrated to produce the new reference phase value for each bunch. Finally, the dc component is removed and the new cavity voltage reference is set. These values are calculated in real time (with a delay of two turns) and updated every turn. The LLRF cannot directly adjust the cavity voltage, but instead modifies the cavity reference and counts on the strong rf feedback and OTFB to impose this on the cavity. These feedback loops have a time constant of about $1 \mu \mathrm{s}$.

The algorithm is implemented in the digital part of the LHC LLRF field-programmable gate array (FPGA). There 
is one LLRF system per cavity. The phase modulation is calculated independently for each cavity. To ensure synchronization between the algorithm and the beam, each LLRF system receives a revolution frequency reference.

It should be noted that the algorithm only relies on the measured $I_{g}$ and $V$ signals and does not require prior knowledge of the beam pattern or bunch intensity. More details on the technical implementation can be found in Appendix A.

\section{SIMULATIONS}

The algorithm was first tested in simulations developed in Simulink. These simulations included models of the rf, beam, cavity, cavity tuner, and LLRF feedback, with settings corresponding to the nominal LHC beam. Only half of the ring was filled with bunches in this simulation to increase the final peak-to-peak phase variation and better test the algorithm's precision.

The adaptive algorithm was tested in two stages. First, an ideal SiMULINK scheme of the whole chain was developed for initial debugging, filter optimization, and testing. This initial model also allowed the tuning of algorithm time constants. The final simulation results were very promising. Figure 6 shows the cavity reference and cavity phase after 4000 simulated turns (a time constant much faster than intended in operation was used in simulations to reduce computational time). The algorithm converged to the expected theoretical values.

In a second stage, the actual firmware was tested. It was inserted into the SIMULINK scheme as a fully functional block. Debugging and partial commissioning was possible with these simulations without valuable LHC time. A lot of safety features were added at that time too, to prevent

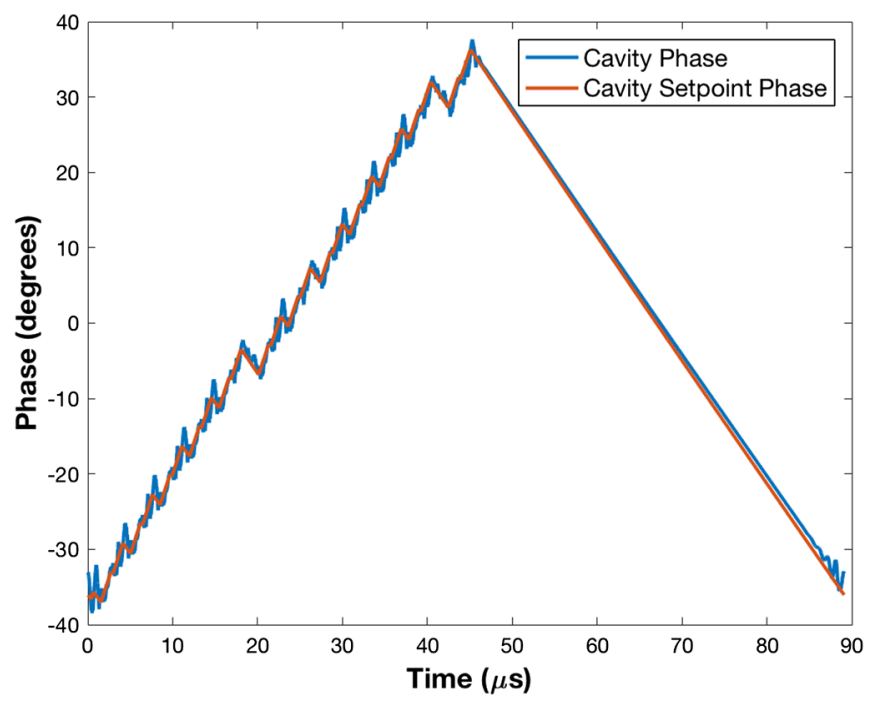

FIG. 6. Simulation of the algorithm with half-full ring. Optimal phase modulation.

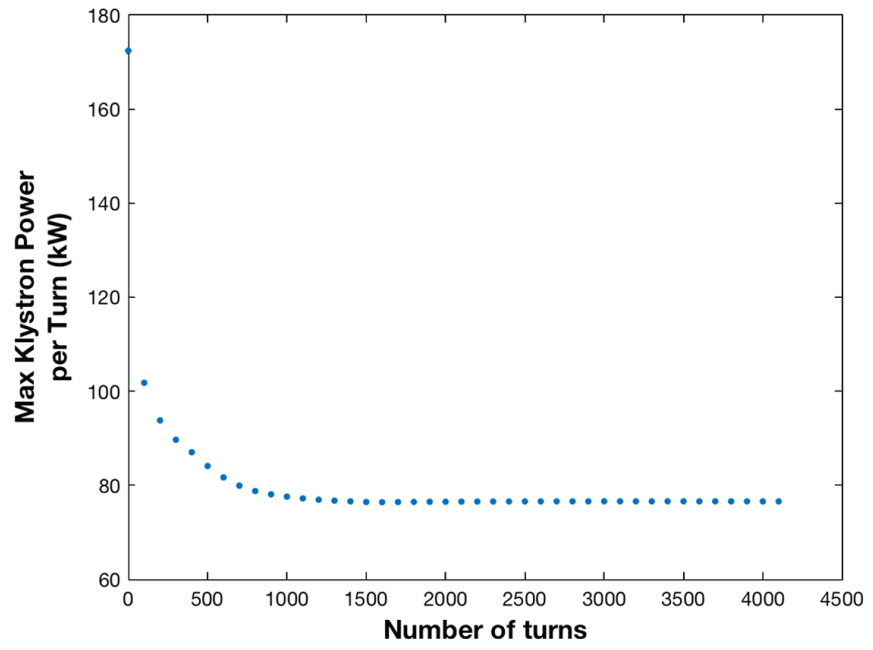

FIG. 7. Peak power reduction as the algorithm optimizes the phase modulation.

the algorithm from imposing a big step to the cavity (and thus beam) phase. The phase variation has to be adiabatic, which requires slow transitions since the LHC synchrotron frequency ranges from $55 \mathrm{~Hz}(450 \mathrm{GeV})$ to $20 \mathrm{~Hz}(6.5 \mathrm{TeV})$.

Figure 7 shows the maximum klystron power over a turn, caused by the transitions between the beam and no-beam segments. The peak power is reduced from $170 \mathrm{~kW}$ to $\approx 75 \mathrm{~kW}$ when the algorithm is switched on, in good agreement with Eq. (1). The reduction of the peak power transients is evident compared to Fig. 1. In fact, as the algorithm converges the power transients disappear and the required power becomes flat.

\section{LHC TEST}

\section{A. Experimental conditions}

An LHC test was performed with nominal LHC conditions at $450 \mathrm{GeV}$ followed by an acceleration ramp to 6.5 TeV [13]. After the initial 12 bunches, batches of 96 bunches were injected up to 1164 bunches for LHC's beam 2 . Due to transfer line issues during the test, only two 96-bunch batches were injected in ring 1 for a total of 204 bunches. The detectors were off during this initial test for safety purposes.

The klystron transient behavior depends on the length of the beam/no-beam segments in the machine. Therefore, the two batch configuration ( $\approx 5 \mu$ s long) for beam 1 in the machine closely resembles the situation of a full machine with an abort gap, whose length is approximately $4.4 \mu \mathrm{s}$. On the other hand, a half-full machine leads to the highest phase modulation along a turn, so the beam 2 pattern provided very useful information too.

The adaptive algorithm was tested during the LHC test. The gain of the algorithm was adjusted so that the time constant was set to about 30 seconds. 


\section{B. Observations}

The primary metric for the algorithm's performance is the average and peak klystron power. It is also important to check that there are no unwanted effects due to the algorithm's action, such as bunch lengthening or beam loss resulting from rf noise produced by the phase manipulations. The phase modulation should be compared among all cavities to confirm that there is no energy exchange between cavities through the beam. The derivation in Sec. II assumes that all cavities are aligned.

\section{Average klystron power}

The average klystron power was significantly reduced for both beams, as expected. Figure 8 shows the average klystron power for all beam 1 stations. The increase in power after the first injection of a long batch is clear at $00: 26$. Injections took place with fixed voltage and phase reference along the ring. It should be noted that the required power does not further increase with subsequent injections since the cavity tuning loop uses a $1 \mu$ s running average of the klystron power, so it settles to the half-detuning value after one long batch. The voltage phase modulation algorithm is switched on at 00:32 and the average klystron power is significantly reduced. The algorithm's gain was increased at $00: 34$, with a further marginal improvement in klystron power, which returns to the preinjection (no beam) level, as expected from the theoretical derivation in Sec. II. The spread in klystron power over all the stations is due to small differences in the cavity fine-tuning and possibly some small drifts in each LLRF system. The average power over all stations is $76.9 \mathrm{~kW}$, compared to the theoretical vale of $77.2 \mathrm{~kW}$ for the desired injection $Q_{L}=20000$. This small discrepancy is reflecting a low precision on the LHC $Q_{L}$ settings and power calibration $(\approx 5 \%)$. The $Q_{L}$ depends on the position of the movable couplers, which are set with an $\approx 10 \%$ error margin. Therefore, there is a significant spread on the $Q_{L}$ values and final power levels for each station, leading to a discrepancy between the theoretical

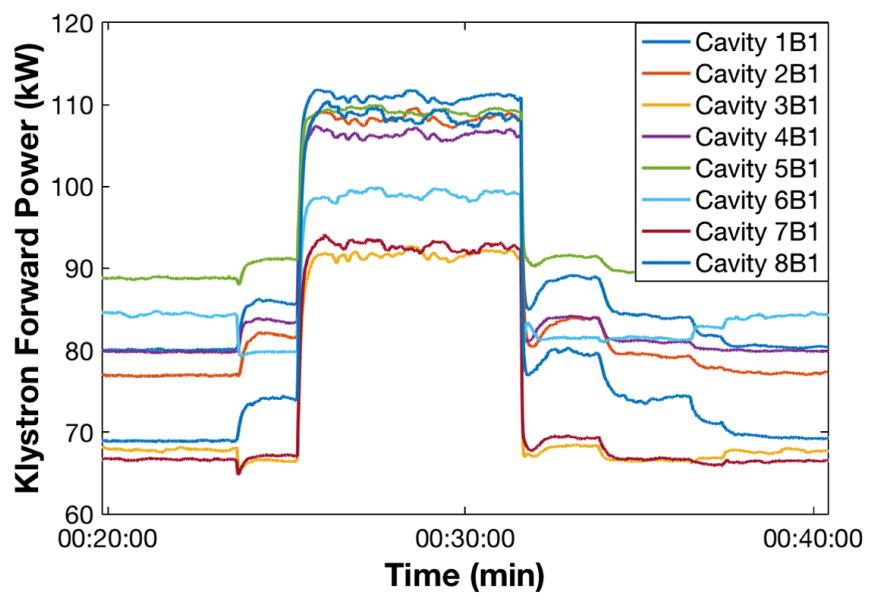

FIG. 8. Average klystron forward power. 204 bunches, beam 1.

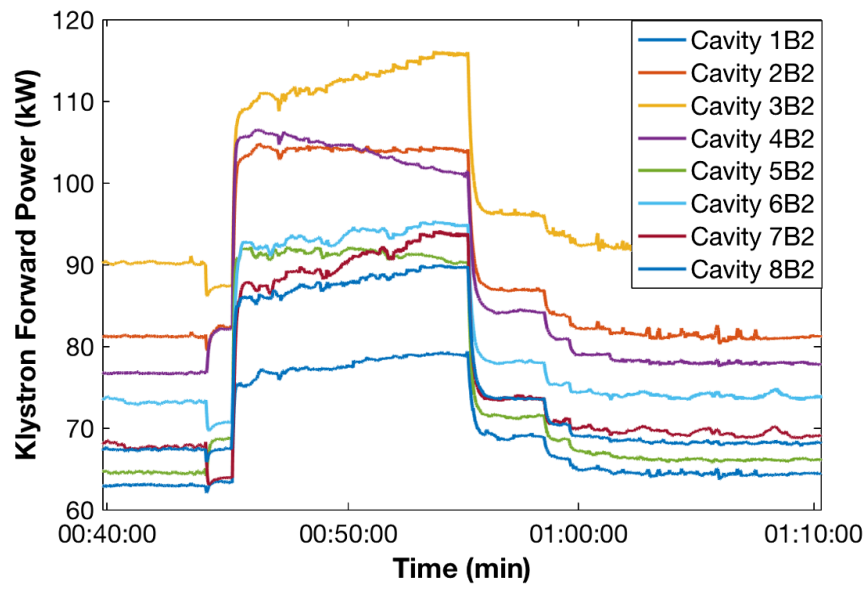

FIG. 9. Average klystron forward power. 1164 bunches, beam 2 .

and average measured power level. Due to the nature of the algorithm though, this power level is indeed the minimum for each station.

Similarly, Fig. 9 shows the average klystron power for all beam 2 stations. The first injection of a 96-bunch batch was at 00:46. Additional batches were injected up to 1164 bunches. The algorithm was switched on at 00:55 with a significant improvement in klystron power requirements. The algorithm's gain was increased at 00:58 and 00:59 with additional (but smaller) gains in performance. The average power over all stations is $74.0 \mathrm{~kW}$, compared to the theoretical optimal value of $77.2 \mathrm{~kW}$. The $4 \%$ error is within the measurement precision described above.

The two beams were then accelerated to $6.5 \mathrm{TeV}$ with the adaptive algorithm on during acceleration. Several $\mathrm{rf}$ parameters change during the ramp: the cavity loaded $Q_{L}$ is increased from 20000 to 60000 in four discrete steps at the

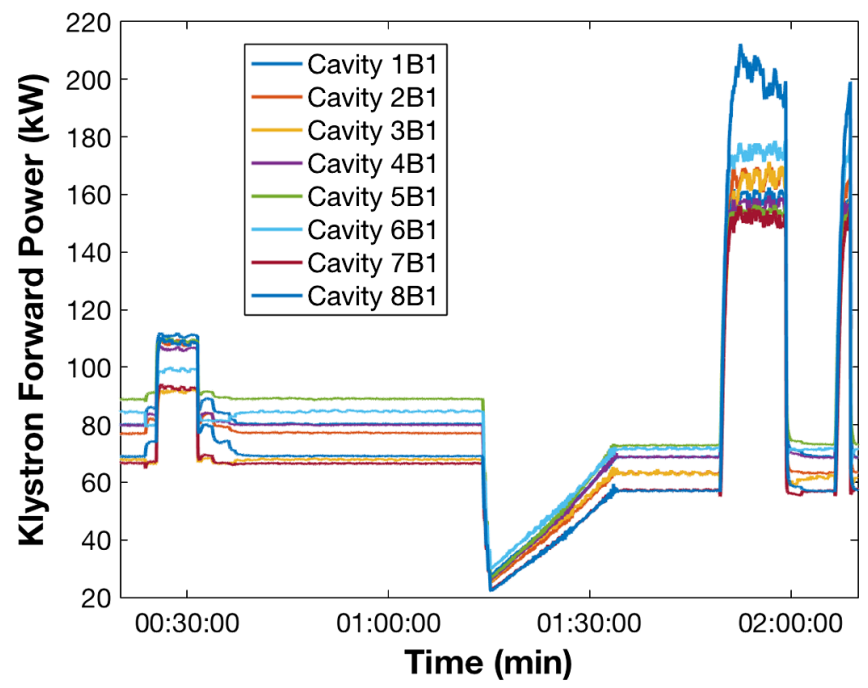

FIG. 10. Average klystron forward power. 204 bunches, beam 1 . 


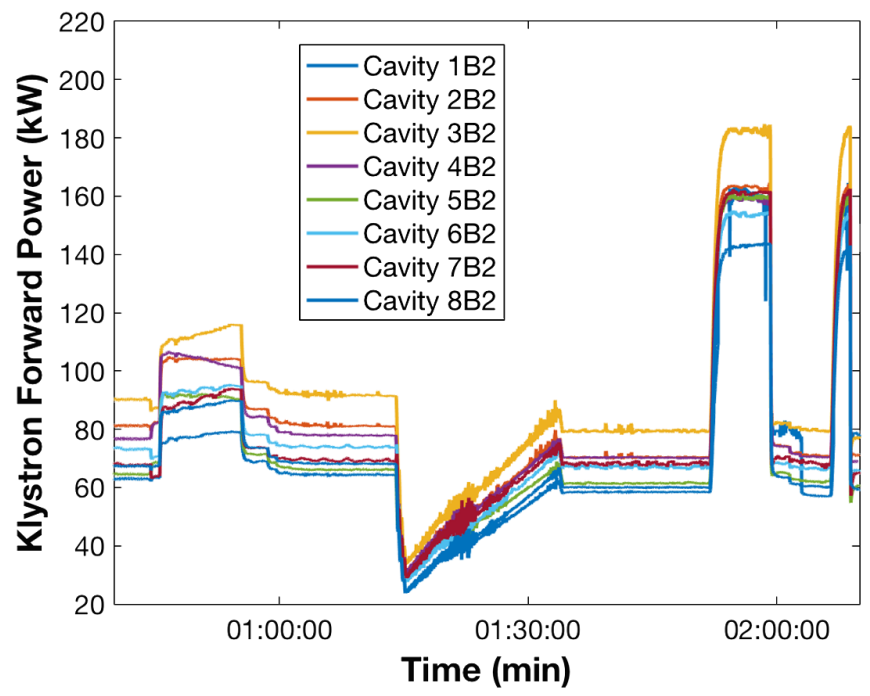

FIG. 11. Average klystron forward power. 1164 bunches, beam 2 .

start of the ramp, the rf voltage increases linearly during the ramp from $0.750 \mathrm{kV}$ to $1.25 \mathrm{MV}$ (per cavity), the stable phase varies between $180^{\circ}$ and $175^{\circ}$ as a consequence of voltage and energy changes. The algorithm automatically adapts to these changes. Figures 10 and 11 show the same time interval as above, but further include the subsequent acceleration ramp (01:15-01:34). The average klystron power is reduced before the start of the ramp, when the $Q_{L}$ value is increased threefold at $01: 13$. The power is then steadily increased during the ramp as the cavity voltage rises. The algorithm was on throughout this process and actively adjusted the optimal cavity phase modulation. The algorithm was switched off at $01: 50$ for beam 1 and $01: 52$ for beam 2 . The required klystron power increased from an average of about $65 \mathrm{~kW}$ to more than $160 \mathrm{~kW}$ for beam 1 and from about 68 to $160 \mathrm{~kW}$ for beam 2 . The algorithm was subsequently switched on again at 01:59 and the performance improvement was achieved again. The algorithm was switched off/on once a last time a few minutes later. All these manipulations were done adiabatically (time constants in the order of 30 seconds).

At $6.5 \mathrm{TeV}$, the average power over all stations was $65.2 \mathrm{~kW}$ for beam 1 and $68.3 \mathrm{~kW}$ for beam 2 , whereas the theoretical optimal value is $72.3 \mathrm{~kW}$ for a $Q_{L}=60000$. The results are very positive. The $10 \%$ and $6 \%$ errors are within the measurement precision described above.

\section{Klystron power per turn}

When it comes to transient beam loading compensation, the most relevant metric is the peak klystron power over a turn. It is thus essential to compare the klystron power per turn with and without the algorithm. With the present fixed cavity phase method ("half detuning") the average klystron power is indeed equal during the beam and nobeam segments $[1,2]$. This results in a high average power.
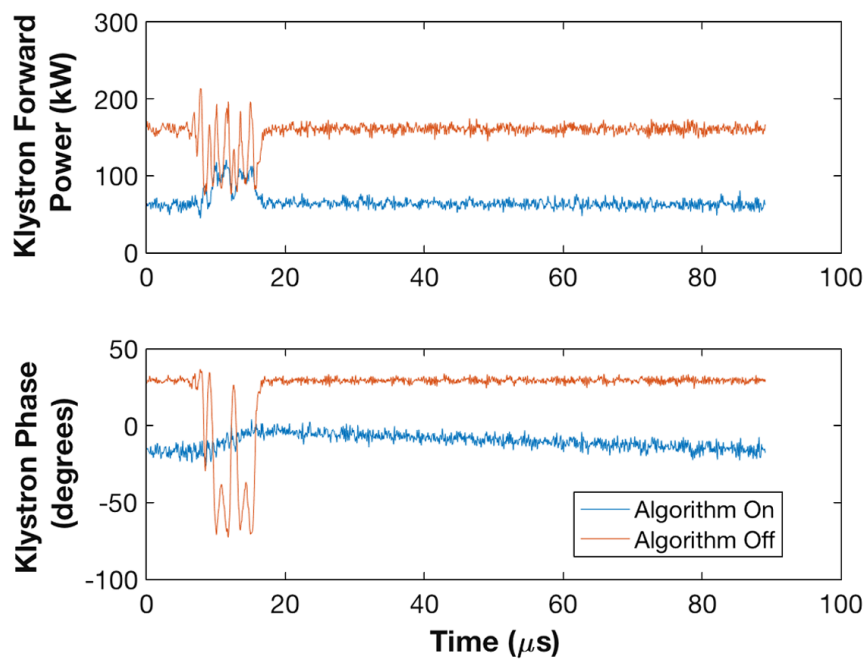

FIG. 12. Klystron forward power per turn. 204 bunches, beam 1 .

The regulation also calls for very large power transients during the beam to no-beam transitions (red traces, Figs. 12 and 13). With the new method (cavity voltage phase modulation and full detuning), the average klystron power during the beam and no-beam segments is still the same, but is now significantly reduced (blue traces). In addition, the peak transients at each beam-no-beam transition (and vice versa) that were observed with the previous method, have now almost disappeared and are within the acquisition noise for beam 2 .

The improvement for beam 1 is significant, but there is still some residual additional power in the beam segment (Fig. 12). The discrepancy was later traced to a phase misalignment between the digital and analog parts of the feedback in the LLRF. After optimizing the LLRF
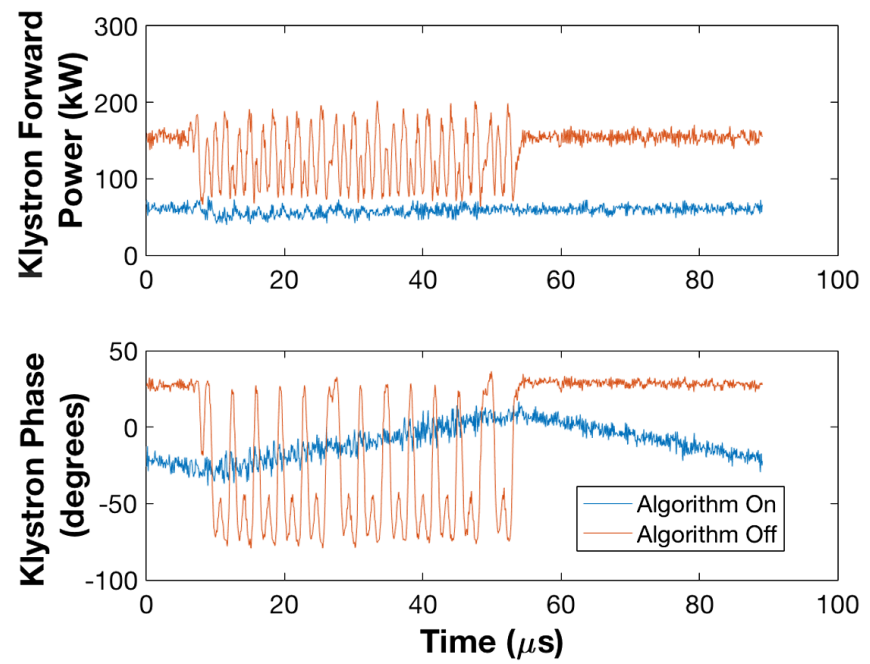

FIG. 13. Klystron forward power per turn. 1164 bunches, beam 2 . 


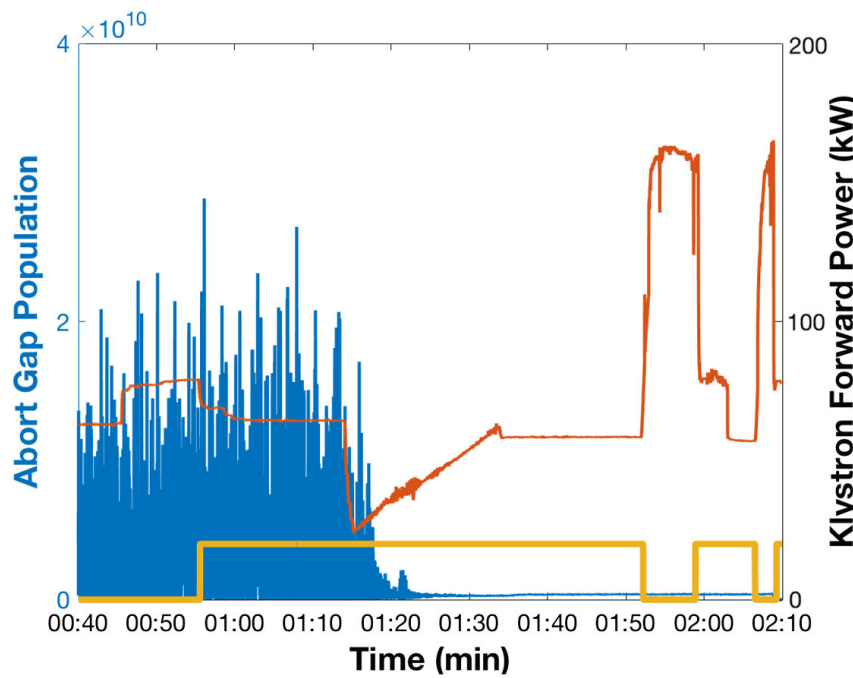

FIG. 14. Beam 2 abort gap population and klystron 1B2 forward power. The yellow line indicates when the algorithm is on/off. The blue line shows the abort gap population and the red line the klystron power.

parameters, the beam 1 algorithm performance is at the same level as for beam 2 .

\section{E. Abort gap and bunch length}

The LHC beam abort kicker rise time is $3 \mu \mathrm{s}$. As a result, it is mandatory to keep an abort gap of at least $3 \mu \mathrm{s}$. Particles that escape the longitudinal bucket slowly make their way to the abort gap.

The abort gap population and bunch length were monitored during the LHC test. There were no signs of additional bunch lengthening nor of an increase in the abort gap population when the algorithm was on, as shown in Figs. 14 and 15. In addition, no debunching was observed

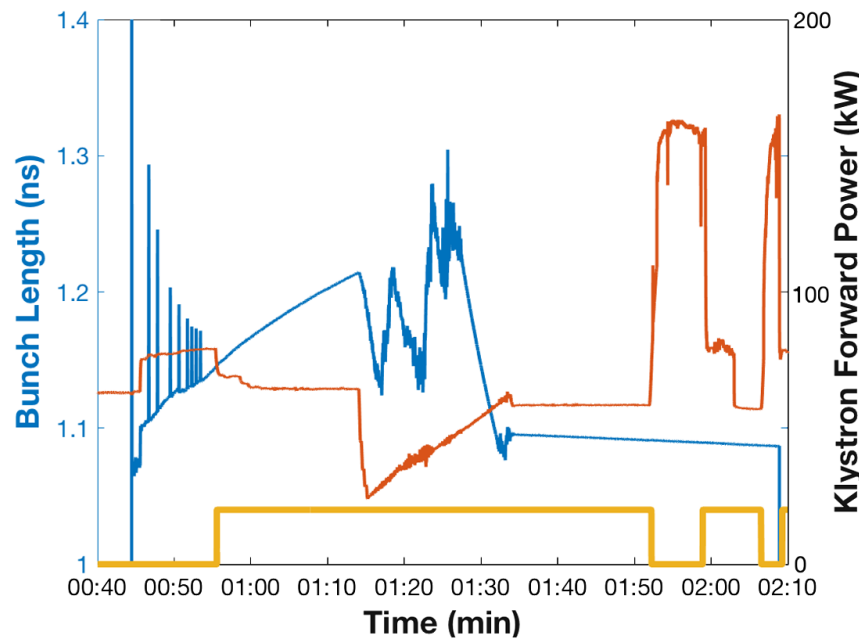

FIG. 15. Beam 2 bunch length and klystron 1B2 forward power. The yellow line indicates when the algorithm is on/off. The blue line shows the bunch length and the red line the klystron power.

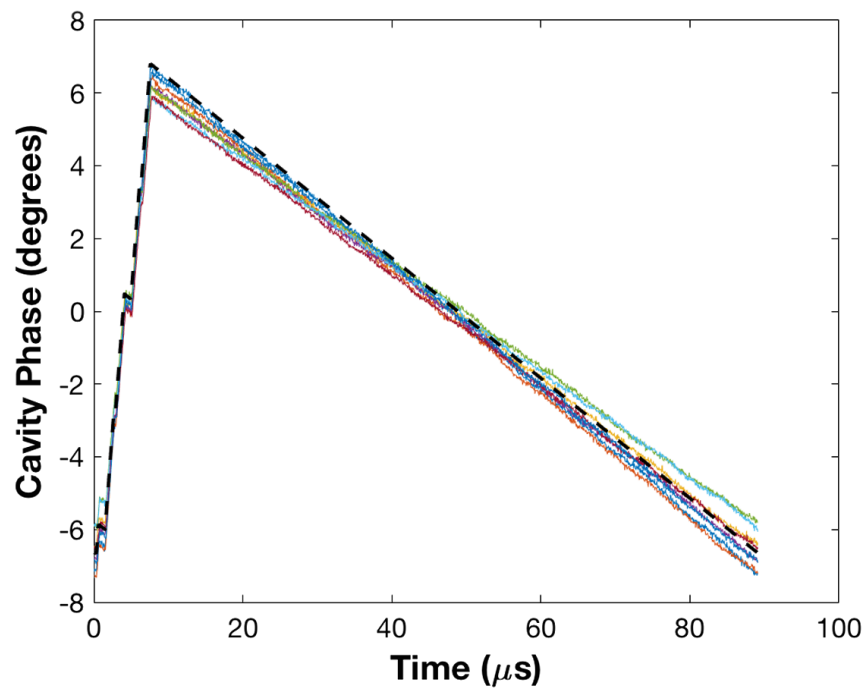

FIG. 16. Phase modulation for all cavities. 204 bunches, beam 1.

when the algorithm was switched on-off and off-on at $6.5 \mathrm{TeV}$.

\section{F. Phase modulation for all cavities}

Figure 16 shows the phase modulation for each cavity at 6.5 TeV. The phase modulation is almost identical for all stations. The black dashed line shows the theoretically estimated optimal phase modulation from Eq. (4), which shows very good agreement with the acquired data. The small differences are due to slightly different LLRF settings for each station (mostly the fine adjustment of the cavity detuning). Figure 17 shows a similar picture for beam 2 . The phase modulation is very similar across all cavities. With the beam intensity found during the LHC test, the

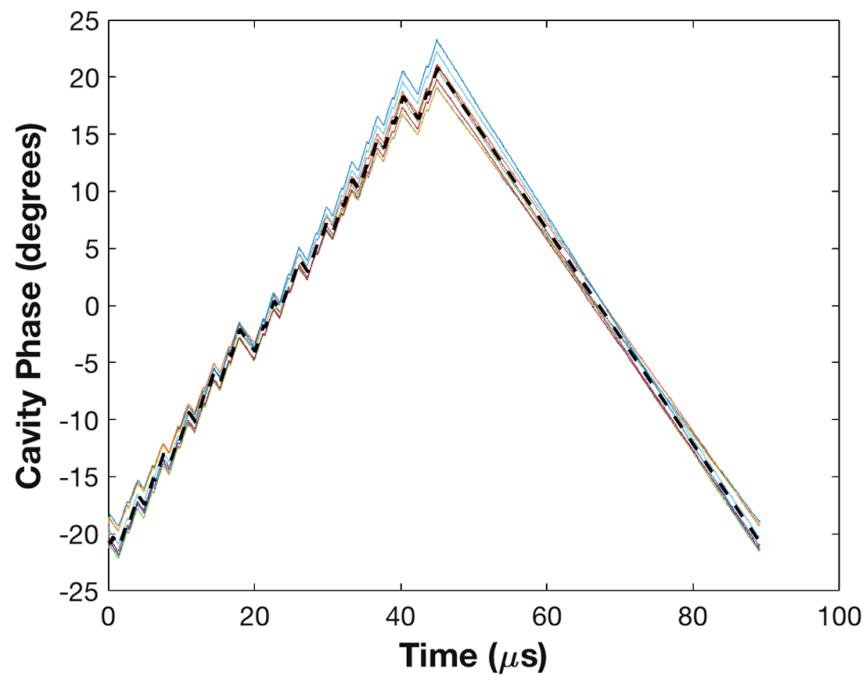

FIG. 17. Phase modulation for all cavities. 1164 bunches, beam 2 . 
theoretical phase modulation is $13.5^{\circ}$ and $41.9^{\circ}$ for beams 1 and 2 respectively, to be compared to the observed $12.1^{\circ}-14.1^{\circ}$ and $38.6^{\circ}-45.5^{\circ}$ over all cavities. The sawtooth modulation along the beam part should be noted: the larger drops are due to the gaps between the 96-bunch super proton synchrotron (SPS) batches (900 ns), the smaller drops correspond to the gaps between the 48-bunch proton synchrotron (PS) batches (225 ns). There are also two $2.1 \mu \mathrm{s}$ long gaps.

\section{LHC TEST WITH DETECTOR DATA COLLECTION}

After the success of the LHC test, a second test was performed at $6.5 \mathrm{TeV}$ during a normal LHC fill with the detectors active. The objective was to see whether the cavity (and thus beam) phase modulation would be an issue for the LHC experiments leading to reduced luminosity. The test was performed after ten hours of physics at 6.5 TeV. As a result, the proton population had dropped to about $0.7 \times 10^{11}$ per bunch. The mean bunch length was at about $1 \mathrm{~ns}$. Each LHC ring had 2220 bunches. The algorithm was left on for about 2 hours.

Since the beam pattern is almost identical for the two rings, the phase modulation should be very similar between the two rings. As a result, no longitudinal shift of the collision point is expected for interaction points 1 (ATLAS detector) and 5 (CMS detector) [11]. On the other hand, the collision time would be modulated over a turn for those two detectors by about 44 ps peak to peak, as calculated by Eqs. (3) and (4). This is of course insignificant compared to the $1 \mathrm{~ns}$ bunch length.

The other two LHC detectors (ALICE, at point 2 and $\mathrm{LHCb}$, at point 8) would see a longitudinal shift of the collision point (about $4.5 \mathrm{~mm}$ peak to peak). The collision time would be modulated by about 34 ps peak to peak for

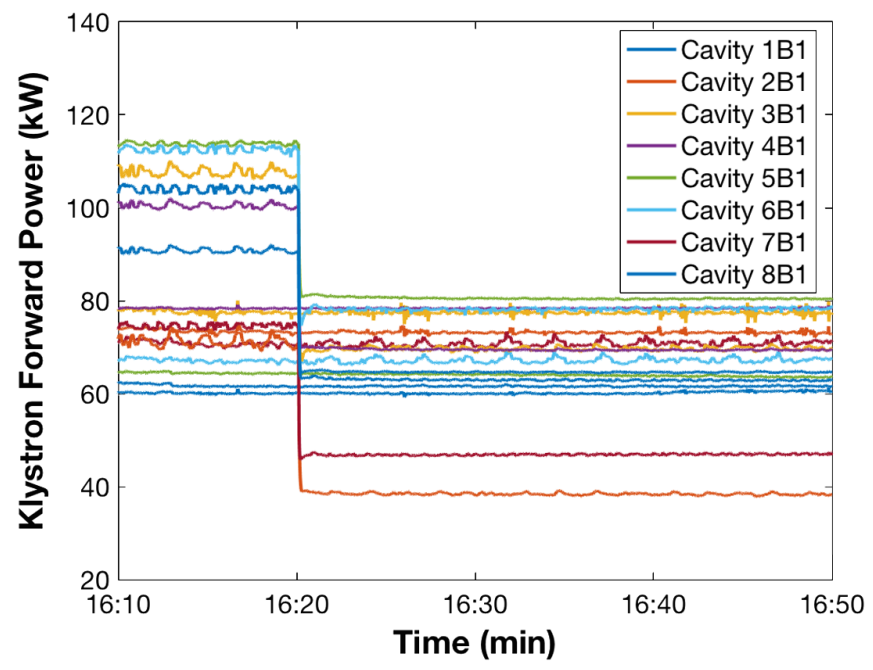

FIG. 18. Average klystron forward power. 2220 bunches, beam 1 .

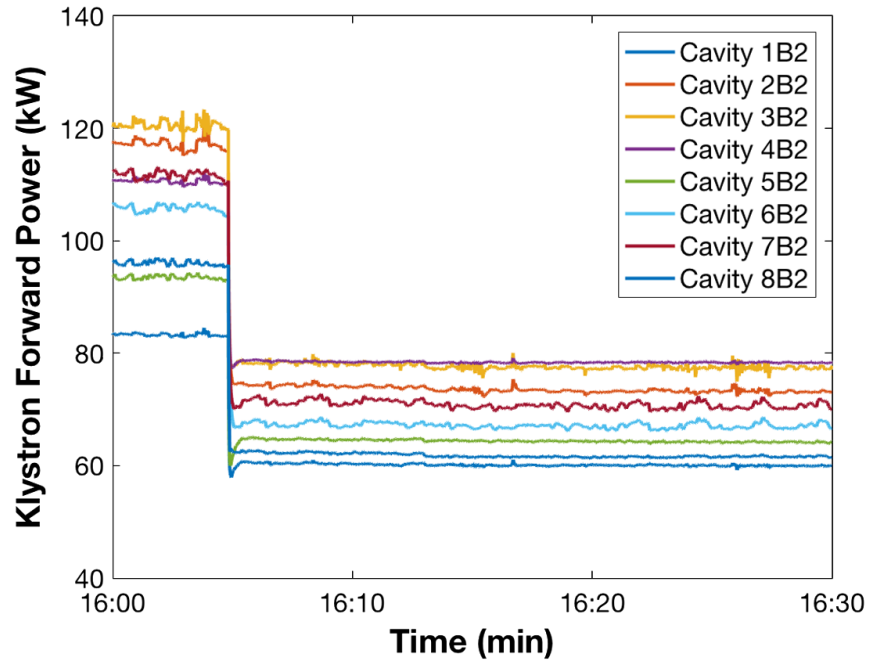

FIG. 19. Average klystron forward power. 2220 bunches, beam 2 .

nominal LHC parameters. Again, these values are very small compared to the bunch length $(1 \mathrm{~ns}, 30 \mathrm{~cm})$.

\section{A. Average klystron power}

The algorithm was switched on at 16:05 for beam 2 and 16:20 for beam 1. The average klystron power was significantly reduced. For beam 1 the final value was in the range of 38-81 kW (Fig. 18) and for beam 2 in the range of $60-79 \mathrm{~kW}$ (Fig. 19). The theoretical estimate is $72.3 \mathrm{~kW}$. As mentioned above, the range of values reflects a cavity detuning spread around the desired value. The algorithm once again successfully reduced the klystron power. No abort gap population increase or bunch lengthening was observed even after two hours of operation with the algorithm on.

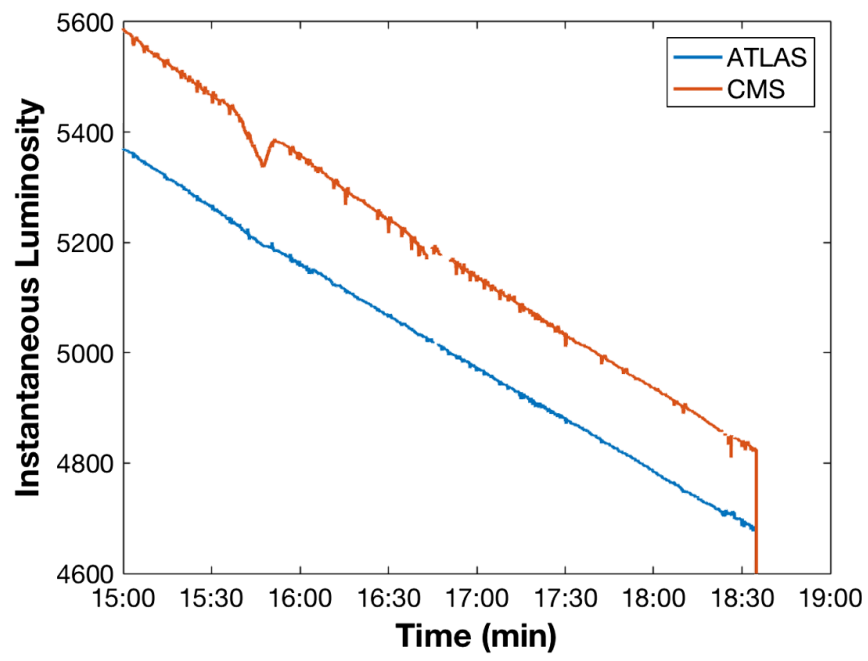

FIG. 20. ATLAS and CMS instantaneous luminosity. 


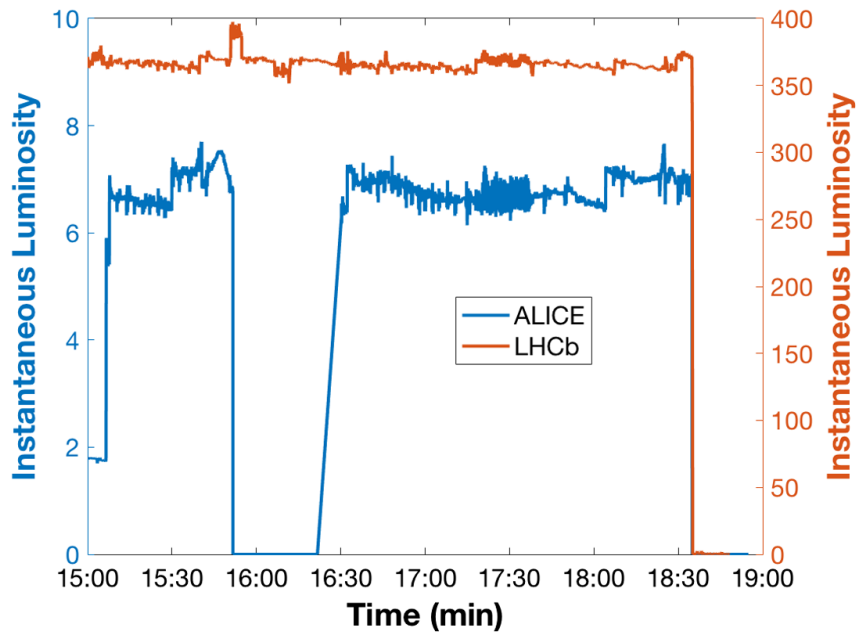

FIG. 21. ALICE and LHCb instantaneous luminosity. ALICE was switched off during the algorithm deployment (15:50-16:20).

\section{B. Luminosity}

Figures 20 and 21 show the instantaneous luminosity from the four LHC experiments during this test. It is clear that the algorithm activation (16:05 and 16:20) had no effect on the luminosity. The algorithm remained on for the remainder of this physics fill. Luminosity scans have been removed to increase clarity. These figures confirm that the very small shifts of the collision point in longitudinal position and timing are insignificant compared to the $1 \mathrm{~ns}$ length of the LHC bunches so there is no luminosity loss.

\section{ATLAS data}

Even though there was no negative effect on luminosity, it is interesting to check whether the measured time and position shifts of the collision point agree with the expected values. Figure 22 shows the collision point time shift for the

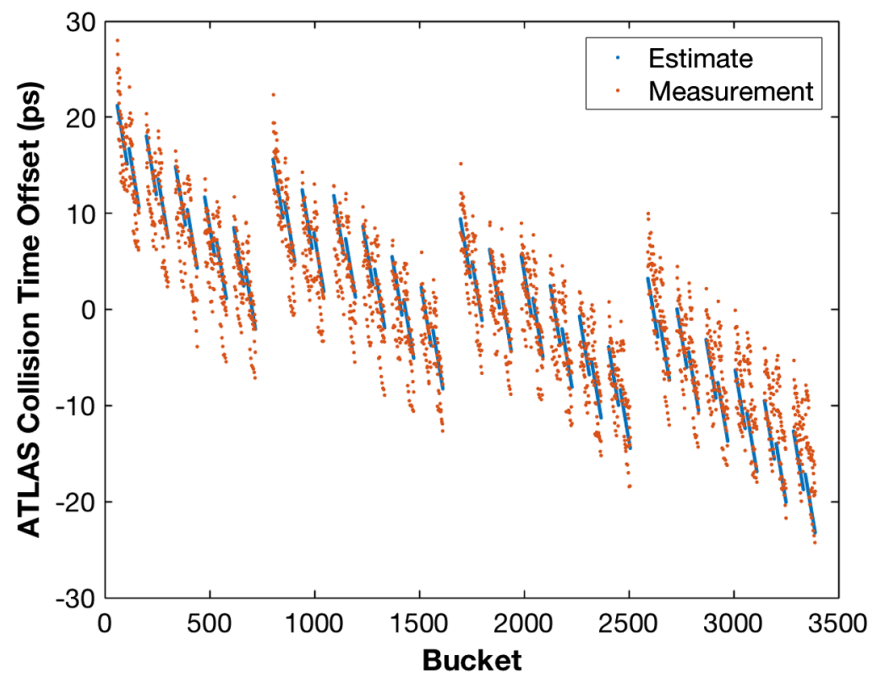

FIG. 22. Collision point time shift for the ATLAS detector.

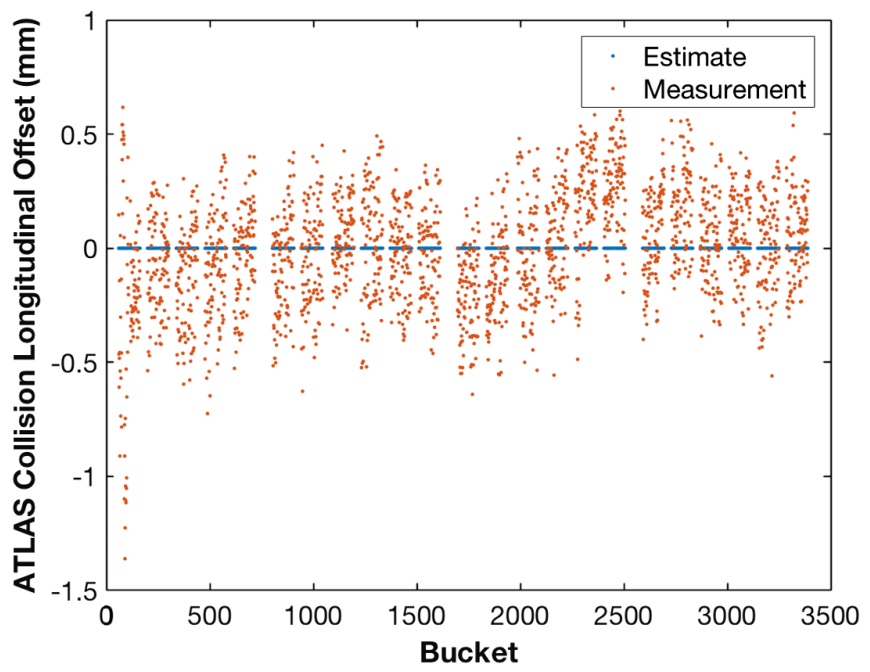

FIG. 23. Collision point longitudinal shift for ATLAS. Data and theoretical estimation.

ATLAS detector. The measurement for each bucket corresponds to the average value over 100 turns, leading to a standard deviation of 6 ps. There is very good agreement with the theoretically estimated shift for the beam and $\mathrm{rf}$ parameters at the time of the test. The estimated value is computed using Eqs. (3) and (4) for both rings. The time shift is then proportional to the average value of the phase shift for each pair of interacting bunches.

The longitudinal position shift should be practically zero for ATLAS due to the symmetry of the interacting beam patterns. Figure 23 shows the measured and estimated position shift. The apparent discrepancy is due to the limited measurement precision (the standard deviation for each bucket is about $0.24 \mathrm{~mm}$ ). The position shift is so small that it cannot be measured.

The CMS detector results are very similar due to the LHC symmetry.

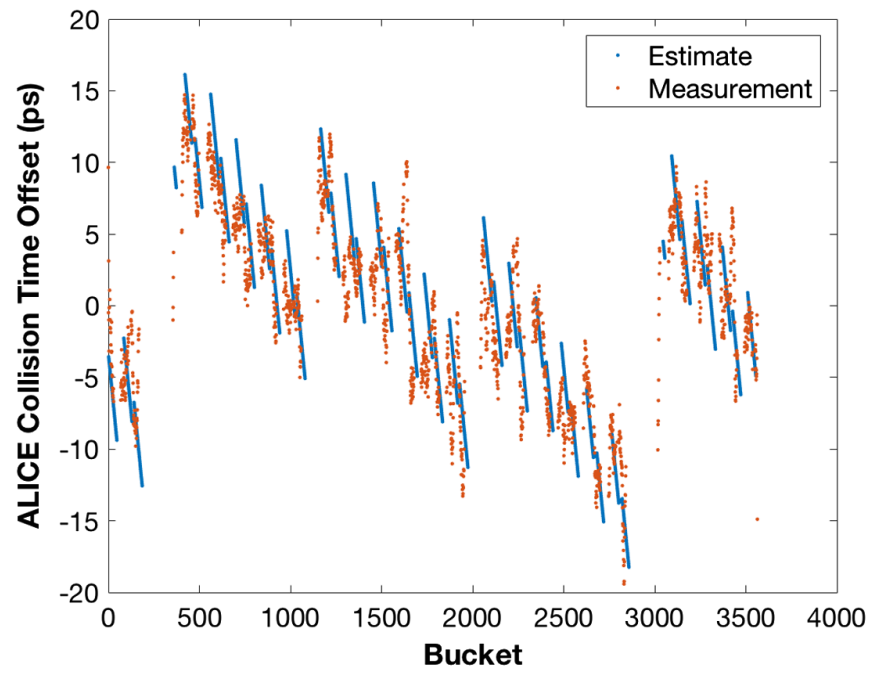

FIG. 24. Collision point time shift for the ALICE detector. 


\section{ALICE data}

The longitudinal position and time shift in the ALICE detector was also investigated. Figure 24 shows the measured and estimated time shift, which show good agreement. The peak-to-peak value is comparable to ATLAS. The longitudinal position shift is higher though, as expected, due to the asymmetry of the interacting beam patterns at Point 2 (Fig. 25). Still though, it is in the order of a few millimeters for a $30-\mathrm{cm}$ long bunch.

\section{HL-LHC PREDICTIONS}

The HiLumi LHC planned operation includes 2748 bunches per beam and $2.2 \times 10^{11}$ protons per bunch, amounting to $1.11 \mathrm{~A}$ dc. The bunch spacing will be $25 \mathrm{~ns}$, the $4 \sigma$ bunch length will be $1 \mathrm{~ns}$, and the abort gap length will be $4.4 \mu \mathrm{s}$.

As can be seen from Eqs. (3) and (4), the phase modulation depends on the beam current, beam pattern, cavity voltage, cavity $R / Q$, and rf frequency. The last two parameters will not change for the HL-LHC, and the beam current should be about $1.1 \mathrm{~A} \mathrm{dc}$. The planned beam pattern (especially the length of the abort gap) and cavity voltage could change though before the HL-LHC starts operating or even during its operation. The bunch length might change as well, which has an indirect effect through the rf component of the beam current (relative bunch form factor [9]).

The LHC test presented in Sec. VI was performed with an abort gap length of $4.4 \mu \mathrm{s}$, a beam current of $0.28 \mathrm{~A} \mathrm{dc}$ (2220 bunches, $0.7 \times 10^{11}$ protons per bunch), an rf voltage of $10 \mathrm{MV}$, and a $4 \sigma$ bunch length of $1 \mathrm{~ns}$. These conditions lead to a peak-to-peak phase modulation of 44 ps.

For HL-LHC operation, the peak-to-peak phase modulation will be about $111 \mathrm{ps}$ (Fig. 5), as will the time shift for

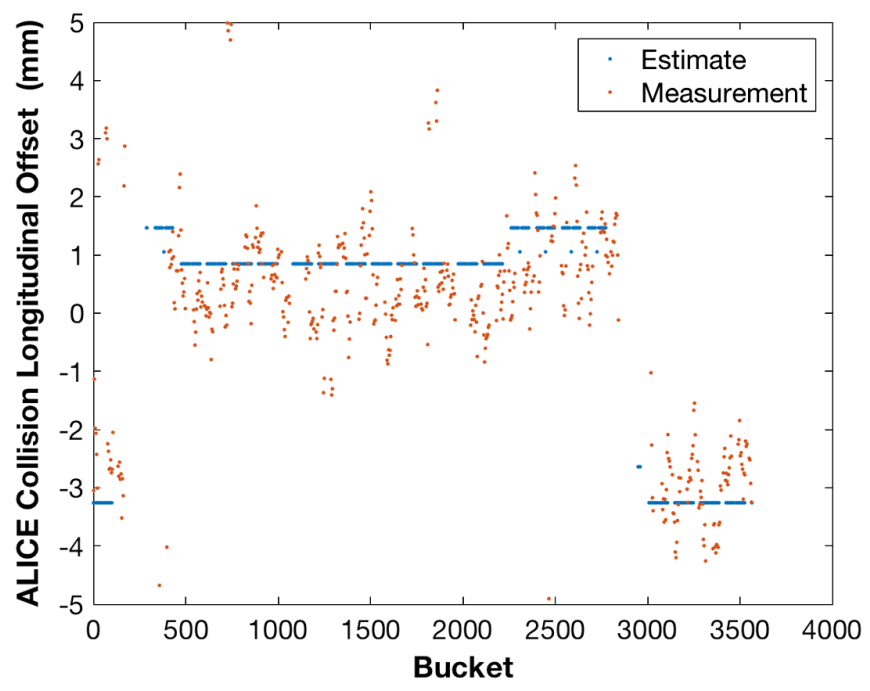

FIG. 25. Collision point longitudinal shift for ALICE. Data and theoretical estimation.
ATLAS/CMS. The time shift for ALICE/LHCb will be about 87 ps. The longitudinal position shift will be about $14 \mathrm{~mm}$ for ALICE/LHCb. Even though the HL-LHC beam current will be about 4 times as high as the LHC test, the cavity voltage will be higher (2 MV instead of $1.25 \mathrm{MV}$ ), leading to just a factor of 2.5 higher phase modulation.

It should be noted that the peak-peak phase modulation increases linearly with the abort gap at constant dc beam current and is inversely proportional to the cavity voltage. The bunch length has a small effect as it changes the bunch form factor: an increase of the $4 \sigma$ bunch length to $1.2 \mathrm{~ns}$ will only reduce the peak-to-peak phase modulation to $103 \mathrm{ps}$.

\section{CONCLUSIONS}

This work presents a cavity phase modulation algorithm for adjusting the cavity reference in anticipation of the beam, thus reducing the klystron power requirements. The optimal phase modulation was derived. The derivation and algorithm could be applicable to other accelerators.

Successful tests in simulations and in the LHC were presented as well. Significant klystron power reduction was observed (peak and average) and the final cavity reference phase modulation approached the theoretically estimated value in these tests.

The algorithm will allow the present LHC rf to deal with the higher intensity beams planned for the coming LHC runs and for the HL-LHC. The algorithm became operational in the LHC on June 4th, 2017, it has been successfully used for LHC physics fills since, and will be used for all LHC runs. As such, it will reduce the required klystron power, slow down the klystron aging, and gain valuable experience before the HL-LHC.

\section{ACKNOWLEDGMENTS}

The authors would like to thank $\mathrm{H}$. Timko for her assistance during the LHC tests, as well as the LHC detectors for the collision point data during the LHC tests, and in particular J. Boyd, C. Schwick, R. Shahoyan, and S. Paramesvaran for facilitating the data delivery. The HiLumi LHC Design Study is included in the High Luminosity LHC project and is partly funded by the European Commission within the Framework Programme 7 Capacities Specific Programme, Grant Agreement No. 284404. This work is also partially supported by the National Science Foundation under Grant No. PHY-1535536.

\section{APPENDIX A: ALGORITHM TECHNICAL IMPLEMENTATION}

The algorithm [Eq. (6)] is implemented in the digital part of the LHC LLRF. The rf signals (generator current and cavity voltage at $400 \mathrm{MHz}$ ) are first mixed with a $380 \mathrm{MHz}$ local oscillator to generate a $20 \mathrm{MHz}$ intermediate frequency (IF) signal. This analogue IF is then sampled with a 
14-bit ADC clocked at $80 \mathrm{MHz}$ to generate in-phase and quadrature (I, Q) pairs at a 40 megasamples per second (MSPS) rate. All the above clocks are phase locked and bunch synchronous (each LLRF receives a revolution frequency train). The 40 MSPS rate also corresponds to the LHC bunch rate (25 ns spacing). The firmware is implemented in an XC4VLX40 FPGA.

To compensate for the cable delays, the generator current vector is aligned in phase and in time with the cavity field signal. This is achieved by a static programmable phase rotator followed by a first in, first out (FIFO) delay with a granularity of one bunch clock.

The generator current is multiplied with the complex conjugate of the measured cavity voltage and the imaginary part of the result is retained. The LHC LLRF cannot directly impose a given cavity voltage. It relies on a strong rf feedback and OTFB to make the cavity voltage almost equal to the desired reference. These feedback loops have a time constant of about $1 \mu \mathrm{s}$, or equivalently, the closed loop response from the cavity reference to the cavity voltage has a bandwidth of $\approx 300 \mathrm{kHz}$. As a result, the algorithm should not attempt to track errors that are outside the cavity field regulation bandwidth (this was confirmed by extensive simulations). The error signal is therefore low-pass filtered via a decimating filter that also reduces the processing rate to 10 MSPS. The resulting signal is appropriately shifted by a programmable delay line (dual port memory) that is responsible for adjusting the overall control loop delay to exactly one LHC turn so that the error signal measured at a given bunch location corrects the voltage for that same bunch.

The dc level (averaged over one turn) is then removed and the loop gain is applied to calculate $\dot{\varphi}$. The resulting values are integrated bunch by bunch with 48 bits width, to avoid truncating the very small error input signal. At the output of the bunch by bunch $\dot{\varphi}$ integrator the topmost 32 bits are selected and integrated to reconstruct the phase $\varphi$. The output of this phase reconstruction integrator is then passed through an additional dc-block filter, to eliminate any remaining $\mathrm{dc}$ offset caused by rounding or truncation errors accumulated over the very long filtering time (tens of seconds). Finally, the signal is interpolated to 40 MSPS, generating a phase-modulated voltage reference via a phase rotator.

\section{APPENDIX B: rf PARAMETERS TABLES}

TABLE I. LHC design rf parameters.

\begin{tabular}{lcccccccc}
\hline \hline$f_{\text {rev }}(\mathrm{Hz})$ & $f_{r f}(\mathrm{MHz})$ & $f_{s}(\mathrm{~Hz})$ & $V_{r f}(\mathrm{MV})$ & $E_{b}(\mathrm{TeV})$ & $\sigma_{\phi}(\mathrm{ns})$ & $I_{b} \mathrm{dc}(\mathrm{A})$ & Bunches & Protons/bunch \\
\hline 11245 & 400.789 & $20-55$ & $6-16$ & $0.45-7$ & 0.25 & 0.55 & 2808 & $1.1 \times 10^{11}$ \\
\hline \hline
\end{tabular}

TABLE II. HL-LHC design rf parameters.

\begin{tabular}{lcccccccc}
\hline \hline$f_{\text {rev }}(\mathrm{Hz})$ & $f_{r f}(\mathrm{MHz})$ & $f_{s}(\mathrm{~Hz})$ & $V_{r f}(\mathrm{MV})$ & $E_{b}(\mathrm{TeV})$ & $\sigma_{\phi}(\mathrm{ns})$ & $I_{b} \mathrm{dc}(\mathrm{A})$ & Bunches & Protons/bunch \\
\hline 11245 & 400.789 & $20-55$ & $6-16$ & $0.45-7$ & 0.25 & 1.1 & 2748 & $2.2 \times 10^{11}$ \\
\hline \hline
\end{tabular}

[1] P. Baudrenghien and T. Mastoridis, Proposal for an rf roadmap towards ultimate intensity in the LHC, in Proceedings of the 3rd International Particle Accelerator Conference, New Orleans, LA, 2012 (IEEE, Piscataway, NJ, 2012).

[2] D. Boussard, in Proceedings of the IEEE 1991 Particle Accelerator Conference (APS Beams Physics) (IEEE, Piscataway, NJ, 1991).

[3] J. Tückmantel, Technical Report No. CERN-AB-2006030, 2006.

[4] J. Tückmantel, Technical Report No. CERN-AB-Note2004-022, 2004.

[5] T. Mastoridis, P. Baudrenghien, A. Butterworth, J. Molendijk, and J. Tückmantel, Technical Report No. CERN-ATS-Note-2012-075 MD, 2012.
[6] T. Mastoridis, P. Baudrenghien, A. Butterworth, J. Molendijk, and J. Tückmantel, Technical Report No. CERN-ATS-Note-2013-013 MD, 2013.

[7] W. Ross, R. Claus, and L. Sapozhnikov, Gap voltage feedforward module for pep-ii low level rf system, in Proceedings of the Particle Accelerator Conference, Vancouver, BC, Canada, 1997 (IEEE, New York, 1997).

[8] The beam current is the part of the beam spectrum that falls within the bandwidth of the cavity with LLRF regulation. In the LHC, this is a $\pm 300 \mathrm{kHz}$ band centered at 400.8 MHz. $I_{b}$ is the beam current after demodulation by the rf frequency. The ratio of the rf Fourier component to twice the dc beam current is defined as the relative bunch form factor [9]. 
[9] J. Tückmantel, Technical Report No. CERN-ATS-Note2011-002 TECH, 2010.

[10] D. Boussard and T.P. R. Linnecar, Technical Reports No. LHC-Project-Report-316 and No. CERN-LHCProject-Report-316, 1999.

[11] The LHC is divided in octants. The LHC points correspond to the eight vertices. The LHC is filled so that the leading bunches for the two beams collide in points 1 and 5 . This filling symmetry leads to a zero longitudinal shift for collisions at those two points.

[12] System Identification (2nd Ed.): Theory for the User, edited by L. Ljung (Prentice Hall PTR, Upper Saddle River, NJ, 1999).

[13] T. Mastoridis, P. Baudrenghien, J. Molendijk, and $\mathrm{H}$. Timko, Technical Report No. CERN-ACC-NOTE-20160061, 2016. 